\title{
CFD ANALYSIS OF FILLING PROCESS FOR A HYDROGEN ENERGY STORAGE SYSTEM
}

\author{
S. Rouhi ${ }^{1 *}$, S. Sadeqi ${ }^{1}$, N. Xiros ${ }^{1}$, J. loup ${ }^{1}$ \\ ${ }^{1}$ University of New Orleans, 2000 Lakeshore Dr, New Orleans, LA, USA.
}

\begin{abstract}
Hydrogen is a clean energy source and can be generated from renewable energy resources [1]. In this research a 3D dynamics simulation for stationary hydrogen storage is performed by using COMSOL Multiphysics computational software. The development of a model according to information collected from different references is described as follows. This time dependent model is based on the Laminar Flow and Heat Transfer in Fluids modules. Laminar Flow is used to compute the velocity and pressure fields for the flow of a single-phase fluid. The equations solved by Laminar Flow are the Navier-Stokes equation for conservation of momentum and the continuity equation for conservation of mass. Heat Transfer solves the Navier-Stokes equation together with an energy balance [15]. In the current model, one node implemented was the Inlet, in which a velocity field was used. The flow direction was assumed to be uniform through the injection pipe in the direction of the cylinder axis. Results show various conditions of velocity and pressure fields along with temperature in different locations inside the storage tube produced through the inflow of hydrogen gas. According to the Bernoulli equation, where the velocity field is zero, the pressure is at maximum value. This fact is seen in the results of this simulation in the right half of the tank where the stagnation plane is created. Zero-dimensional modeling has also been carried out to compare with the results. This model shows qualitative agreement with the COMSOL results.
\end{abstract}

KEY WORDS: Hydrogen storage, Computational methods, COMSOL, Zero-dimensional modeling, Laminar flow, Navier-Stokes equation

\section{INTRODUCTION}

Under normal conditions of pressure and temperature, hydrogen is a colorless, odorless, tasteless, nonpoisonous but flammable gas [2]. The energy density of a component is defined by the product of the energy content and the density of a considered volume particle. It is really a measure of how compactly hydrogen atoms are packed in a fuel [3]. It follows that hydrocarbons of increasing complexity (with more and more hydrogen atoms per molecule) have increasing energy density. At the same time, hydrocarbons of increasing complexity have more and more carbon atoms in each molecule so that these fuels are heavier in absolute terms. On this basis, hydrogen's energy density is poor (since it has such low density) although its energy to weight ratio is the best of all fuels (because it is so light) [3].

Hydrogen can be stored in six different methods as listed below [4]:

(1) high-pressure gas cylinders (up to 800 bar) [4],

(2) liquid hydrogen in cryogenic tanks (at $21 \mathrm{~K}$ ) [4],

(3) adsorbed hydrogen on materials with a large specific surface area adsorbents (at $\mathrm{T}<100 \mathrm{~K}$ ) [4],

(4) absorbed on interstitial sites in a host metal (at ambient pressure and temperature) [4],

*Corresponding Author: srouhi@uno.edu 
(5) chemically bonded in covalent and ionic compounds (at ambient pressure) [4], and

(6) through oxidation of reactive metals, e.g. $\mathrm{Li}, \mathrm{Na}, \mathrm{Mg}, \mathrm{Al}, \mathrm{Zn}$ with water [4].

Most of the research that has been done on hydrogen storage is based on how to store hydrogen for the mobile application as a compact and lightweight energy carrier. The storage of hydrogen is challenging. Being the lightest molecule, hydrogen gas has a very low density: $1 \mathrm{~kg}$ of hydrogen gas occupies over $11 \mathrm{~m}^{3}$ at room temperature and atmospheric pressure. Thus, for the storage of hydrogen to be economically viable, its storage density must be increased. Several methods to store hydrogen at increased density exist. However, all these methods require some input of energy in the form of work, heat, or, in some cases, hydrogen-binding materials. [5], [6].

The most common storage system is the use of high pressure gas steel cylinders, which are operated at a maximum pressure of 200 bar. Depending on the tensile strength of the cylinder material, higher pressures can be reached. New lightweight composite cylinders have been developed that are able to withstand pressure up to $800 \mathrm{bar}$, so that hydrogen can reach a volumetric density of $40 \mathrm{~kg} / \mathrm{m}^{3}$, almost half as much in its liquid form at the normal boiling point. Hydrogen density does not follow a linear function over the increase of pressure. A hydrogen density of $20 \mathrm{~kg} / \mathrm{m}^{3}$ is reached at 300 bars. The volumetric density can be increased to around 36 up to $70 \mathrm{~kg} / \mathrm{m}^{3}$ by compressing the gas to a pressure of up to 700 or 2000 bar, respectively. However, 2000 bar is technically not feasible [7], [8].

Stationary hydrogen storage is a very important part of the hydrogen infrastructure, required for transport and power generation applications. Bulk hydrogen storage facilities are necessary elements of hydrogen production plants, regional/local hydrogen distribution centers and end-use sites, such as refueling stations or fuel cell-based power generation plants. The requirements for storage capacity vary with the application; storage capacities may range from thousands of tons in production sites, to 50-100 tons in distribution centers, to tens of tons in refueling stations. [9], [11].

The restrictions for stationary hydrogen storage are commonly less than for mobile storage. For example, for most stationary applications the weight requirement is relaxed, although the requirement of volume minimization for the storage vessel is important. Other requirements related to stationary storage vessels include the ability to manufacture these vessels with a range of storage capacity; tolerance of the vessel to poisoning by trace elements, good long-term cycling behavior, reasonably fast recharge and delivery flow rates and low hydrogen losses [12], [13]. Finally, the requirement for low cost vessels for stationary applications is not as stringent as for transport applications. However, production, operating and maintenance costs have to be minimized to make the hydrogen infrastructure competitive with the conventional fuel infrastructure [9].

The ultimate goal of the current research is to obtain a comprehensive and practical model by confirming the pressure gradients mathematical model with computational fluid dynamic model. At that point in time, utilize the authenticated model to investigate other parameters behavior throughout the filling procedure to possess explicit idea about how the final state was created. In this manner, this knowledge could be extremely valuable and feasible to develop other alternatives and further tools to manage and adjust the process.

\section{MATHEMATICAL MODEL}

Zero-dimensional analysis is a powerful mathematical tool used to predict physical quantities that change during a specific process. Since there is no spatial dimension involved, it converts a sophisticated process to a simplified model that can help us to have a better insight about the phenomenon. 
Assume that the mass $[m(t)]$, pressure $[P(t)]$, volume $[V(t)]$, and temperature $[T(t)]$ at a given time are known for the control volume. Now when the valve is opened the gas can travel to the storage system, where $\left[\dot{m}_{i}(t)\right]$ denotes inlet mass flow rate. The goal is to find $[m(t+\Delta t)],[P(t+\Delta t)],[V(t+\Delta t)]$, and $[T(t+\Delta t)]$. The volume is not a time variable, therefore it is a constant number through the analysis. The system gains no heat from its surroundings. The continuity equation states that the accumulation of mass into the system is equal to the difference of the mass which enters a system and leaves the system.

$$
\frac{d m}{d t}=\dot{m}_{i}(t)-\dot{m}_{o}(t)
$$

where

$\mathrm{m}$ is the mass $(\mathrm{kg})$

$\dot{m}_{i}$ is the inlet mass flow rate $(\mathrm{kg} / \mathrm{s})$

$\dot{m}_{o}$ is the outlet mass flow rate $(\mathrm{kg} / \mathrm{s})$

And $t$ is the time (s).

where there is no outlet flow $\left\{\dot{m}_{o}(t)=0\right\}$, hence,

$$
m(t+\Delta t)=m(t)+\dot{m}_{i}(t) \cdot \Delta t
$$

By assuming that the system is empty at the time $t$, equation 2 can be simplified as follows

$$
m(t+\Delta t)=\dot{m}_{i}(t) \cdot \Delta t
$$

The control volume allows us to apply the principle of conservation of energy. The pressure at the inlet produces a definite amount of work which pushes the fluid through the system. The modified first law of thermodynamics states that the change of internal energy $[U(t)]$ of the control volume system equals the difference of the net heat transfer $[\mathrm{d} Q(t) / d t]$, the net work is done by the system $[\mathrm{d} W / d t]$ and the enthalpy of the system.

$$
\frac{d U}{d t}=\dot{Q}(t)-\dot{W}(t)+\dot{m}_{i}(t) h_{i}
$$

The change of the internal energy of the ideal gas can be derived from equation 5

$$
U(t)=\mathrm{m}(\mathrm{t}) c_{v} T(t)
$$

where

$c_{v}$ is the amount of heat needed to increase the temperature of the control volume system of the $[1 \mathrm{Kg}]$ mass by $\left[1^{\circ} \mathrm{C}\right]$ temperature.

From the definition of a time change and using equation (5),

$$
U(t+\Delta t)=U(t)+\frac{d U}{d t} \Delta t
$$

Substituting the internal energy into equation (7) to find the temperature

$$
U(t+\Delta t)=m(t+\Delta t) c_{v} T(t+\Delta t)
$$


Consequently we can conclude that

$$
P(t+\Delta t)=\frac{m(t+\Delta t) c_{v} T(t+\Delta t)}{V}
$$

\section{SIMULATION AND STRATEGY}

CFD Module is an optional add-on package for COMSOL Multiphysics that provides tools for computational fluid dynamics, CFD. Once a model is defined, you can go back and make changes in all of the branches listed, while maintaining consistency in the other definitions throughout. Since fluid flow simulations are often computationally demanding, a multistage modeling strategy is usually required. This implies using a simplified model as a starting point in the project. Complexities can then be introduced gradually so that the effect of each refinement of the model description is well understood before introducing new complexities [15].

\section{DEVELOPING THE MODEL}

The development of the model according to the information that is collected from different references is described as follows. However, it is worth pointing out that we simplified the model in many ways to reduce the uncertainties as a result of the modeling strategy which is discussed above.

\section{Geometry:}

The physical size of the stationary hydrogen storage was estimated. Simplifying the geometry reduces the simulation time. We used a $1.4 \mathrm{~m}$ diameter cylinder that was $7.3 \mathrm{~m}$ long with rounded-off corners to simplify the geometry to reduce mesh resolution. However, the curvature of the cylinder is not completely known. The diameter of the injection pipe is $0.2 \mathrm{~m}$. Also, a cylindrical tube is used as an injection pipe.

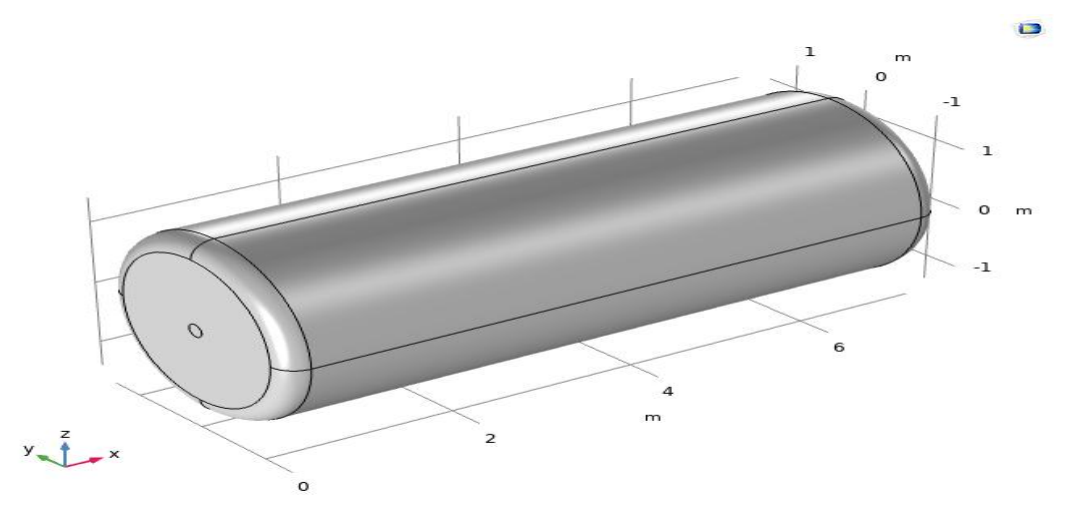

Figure 1. Computational Geometry

\section{Material Properties:}

The hydrogen properties were added from COMSOL's built-in material library.

Defining the Physics Interfaces and Features [15]: 
Different types of flow require different equations to describe them. Due to the fact that for single-phase flow (spf), the Laminar Flow interface is a good place to start, we have simulated our model by combining the Laminar Flow and Heat Transfer in Fluids modules. The Laminar Flow (spf) interface is used to compute the velocity and pressure fields for the flow of a single-phase fluid in the laminar flow regime. The equations solved by the Laminar Flow interface are the Navier-Stokes equations for conservation of momentum and the continuity equation for conservation of mass. The Single-Phase Fluid Flow interfaces are based on the Navier-Stokes equations, which in their most general form read:

$$
\frac{\partial \rho}{\partial t}-\nabla \cdot(\rho u)=0
$$

This equation is known as the continuity equation that represent the law of the conservation of mass. Where

$\rho$ is the density $\left(\mathrm{kg} / \mathrm{m}^{3}\right)$

$\mathrm{u}$ is the velocity vector $(\mathrm{m} / \mathrm{s})$

$\mathrm{t}$ is time (s).

$$
\rho \frac{\partial u}{\partial t}+\rho(u . \nabla) u=\nabla \cdot[-p I+\tau]+F
$$

This equation is a vector equation which identify the law of the conservation of momentum or generally Newton's second law of motion. Where

$\mathrm{P}$ is the pressure $(\mathrm{Pa})$

$\tau$ is the viscous stress tensor $(\mathrm{Pa})$

$\mathrm{F}$ is the volume force vector $\left(\mathrm{N} / \mathrm{m}^{3}\right)$

$$
\rho C_{p}\left(\frac{\partial T}{\partial t}+(u . \nabla) T\right)=-(\nabla . q)+\tau: S-\frac{T}{\rho} \frac{\partial p}{\partial t} \mathrm{I}\left(\frac{\partial p}{\partial t}+(u . \nabla) p\right)+Q
$$

Where

The operation (:) denotes a contraction between tensors which defined as

and

$$
a: b=\sum_{n} \sum_{m} a_{n m} b_{n m}
$$

$\mathrm{P}$ is the pressure $(\mathrm{Pa})$

$\mathrm{F}$ is the volume force vector $\left(\mathrm{N} / \mathrm{m}^{3}\right)$

$C_{p}$ is the specific heat capacity at constant pressure ( $\left./(\mathrm{kg} . \mathrm{K})\right)$

$\mathrm{T}$ is the absolute temperature $(\mathrm{K})$

$\mathrm{q}$ is the heat flux vector $\left(\mathrm{W} / \mathrm{m}^{2}\right)$

$\mathrm{Q}$ contains the heat sources $\left(\mathrm{W} / \mathrm{m}^{2}\right)$

$\mathrm{S}$ is the strain-rate tensor.

The momentum of the compressible flow equation is derived from continuity equation

$$
\rho \frac{\partial u}{\partial t}+\rho(u \cdot \nabla) u=-\nabla p+\nabla \cdot\left(\mu\left(\nabla u+(\nabla u)^{T}\right)\right)-\frac{2}{3} \mu(\nabla \cdot u) I+F
$$

Where 
$\mu$ is the dynamic viscosity $(P a . s)$ that is, for a Newtonian fluid is allowed to depend on the thermodynamic state but not on the velocity field. All gases and many liquids can be considered Newtonian. When the Laminar Flow interface is added, the following default nodes are also added in the Model Builder: Fluid Properties, Wall (the No Slip boundary condition was used), and Initial Values. No slip is the boundary condition to model solid walls. No Slip wall is a wall where the fluid velocity relative to the wall velocity is zero. For a stationary wall that means that $u=0$. Other node that was implemented was the Inlet, in which a velocity field condition was used. The flow direction was assumed to be uniform through the injection pipe in the direction of the cylinder axis.

Heat Transfer interfaces solve the Navier-Stokes equations together with an energy balance. Heat Transfer interfaces can also solve the fully compressible form of the Navier-Stokes equations. It also solves the heat equation, which for a fluid is given by equation 11. The work done by pressure changes is given by the term

$$
\begin{gathered}
\alpha_{p} T\left(\frac{\partial p}{\partial t}+u . \nabla p\right) \\
\text { where } \alpha_{p}=-\frac{1}{\rho} \frac{\partial \rho}{\partial T} \mid p
\end{gathered}
$$

The viscous heating terms $[\tau: S]$ are not included because they are usually negligible. Another node implemented was the Inflow, in which a different temperature boundary condition was used. The heat transfer direction was assumed to be uniform through the injection pipe in the direction of the cylinder axis.

According to the assumptions and then the definition of the Reynolds number which is

$$
R e=\frac{v D}{v}
$$

Where

$\mathrm{V}$ is the velocity of the inlet flow $(\mathrm{m} / \mathrm{s})$

$\mathrm{D}$ is the diameter $(\mathrm{m})$

And $v$ is the kinematic viscosity $\left(\mathrm{m}^{2} / \mathrm{s}\right)$

Also,

$$
v=\frac{\mu}{\rho}
$$

Where,

$\mu$ is the dynamic viscosity (Pa.s) and

$\rho$ is density of the fluid flow $\left(\mathrm{kg} / \mathrm{m}^{3}\right)$.

Kinematic viscosity of the currents case

$v=\frac{\mu}{\rho}=\frac{1.21 \times 10^{-5}}{0.0813}=14.88 \times 10^{-5}\left(\mathrm{~m}^{2} / \mathrm{s}\right)$

And then by considering that $\mathrm{D}=0.2(\mathrm{~m})$ and $\mathrm{v}=1(\mathrm{~m} / \mathrm{s})$

$$
R e=\frac{v D}{v}=\frac{0.2 \times 1}{14.88 \times 10^{-5}}=1344.086
$$

According the the [17], under most practical conditions, the flow in the circular pipe is laminar for 
$R e \ll 2300$. By assuming that in the current research, the Reynolds number is 1344.086 , consequently, the flow is laminar. By focusing on the equation 15, it is clear that the kinmatic viscosity of the hydrogen in the defined temperature of the model is constant due to the density and dynamic viscosity. It has been understood that the boundariy of the laminar flow due to the Reynolds number is less than 2300, the only parameter that could be changed is velocity and diameter in the equation 15 . By attention to these assumptions, diameter and velocity were considred as $0.2(\mathrm{~m})$ and $1(\mathrm{~m} / \mathrm{s})$.

Mesh:

A physics-controlled mesh was implemented in order to reduce numerical diffusion to a minimum. It is crucial to note that to reduce the computational domain and improve time efficiency we used a finer mesh around the edges and boundaries and a coarser one for the simpler smoother part. Figure 2 shows details of the mesh created.

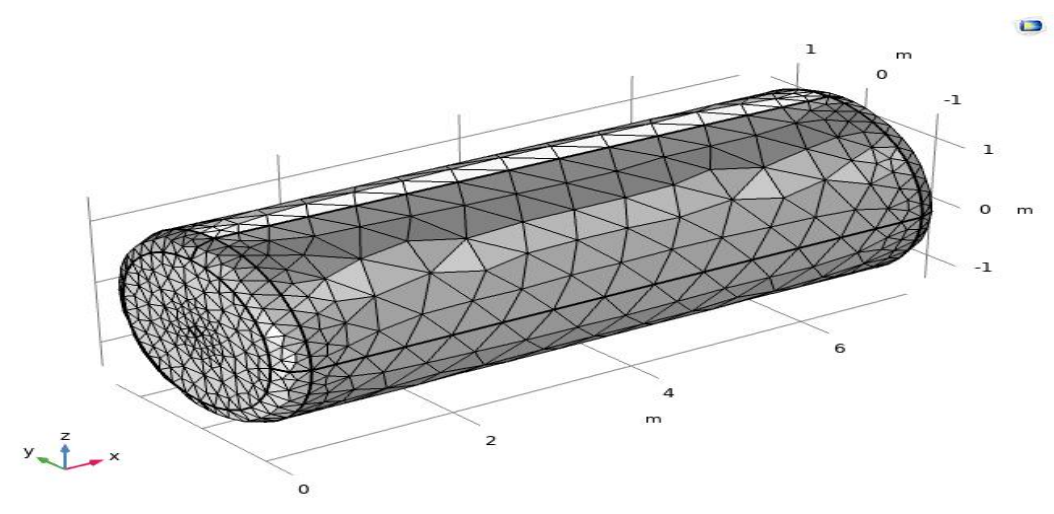

Figure 2. Details of the Mesh

\section{The Choice of Solver and Solver Settings}

The simulation is a time-dependent model with time ranges from zero to three-hundred seconds, with a time step of 0.1 (s). After three-hundred seconds the storage is almost half full because of the 50 bar full capacity of the storage. The restriction for choosing this time was the computer capacity. It is too expensive and time consuming for the longer time simulation. In fact, based on more than ten different time conditions by trial and errors, all of them were diverged, and ultimately, this time interval $(\mathrm{t}=0: 0.1: 300 \mathrm{~s})$ was chosen due to the reasonable convergence.

\section{RESULTS AND DISCUSSION}

Figure 4 provides the pressure field in the gas tank storage after 5 minutes of the filling process at the center of it. 


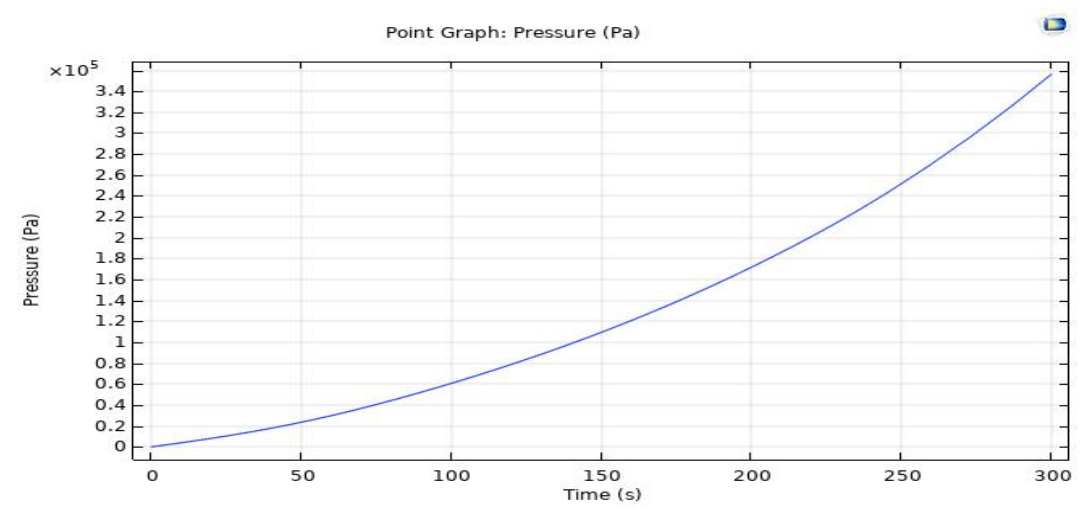

Figure 3. pressure field in the gas tank storage after 5 minutes from COMSOL

The mathematical analysis was implemented in MATLAB to work out the final pressure for the same initial conditions during the same time interval. Figure 4 illustrates the pressure as a function of time.

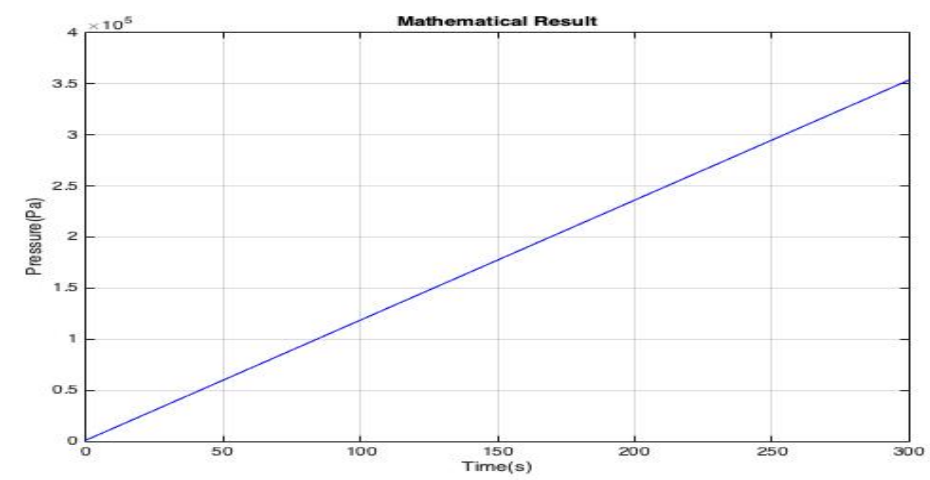

Figure 4. pressure field in the gas tank storage after 5 minutes from mathematical method

According to the fact that the ideal-gas law was assumed for the filling process, the pressure is growing linearly. Figure 5 shows both methods in a same graph for comparison.

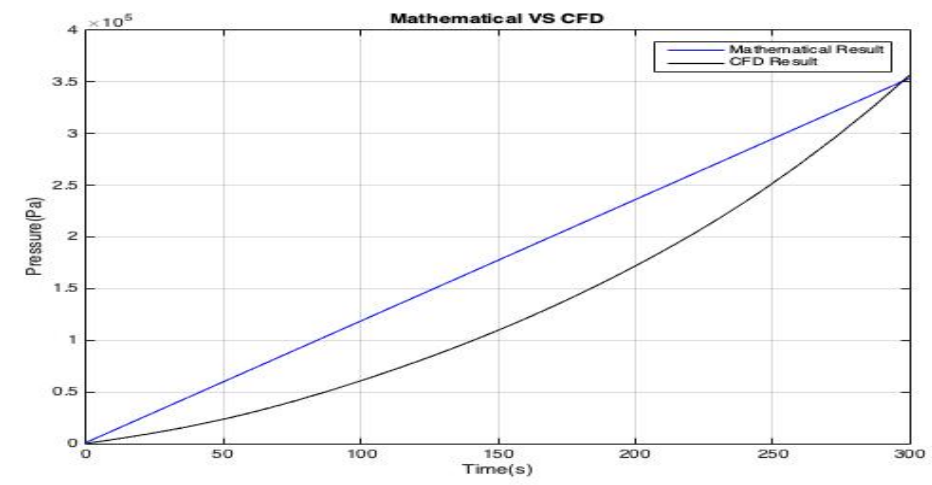

Figure 5. comparison of pressure field in the gas tank storage after 5 minutes 
Althogh, the results show different trending, both methods reached almost the same final pressure. To investigate the pressure more precisely, figure 6 shows the pressure at three cut-lines along the altitude of the cylinder. The blue line represents the line at the center, the green line represents the line at the top of the tank parallel to the height, and the red line represents the line at the bottom of the tank parallel to the height.

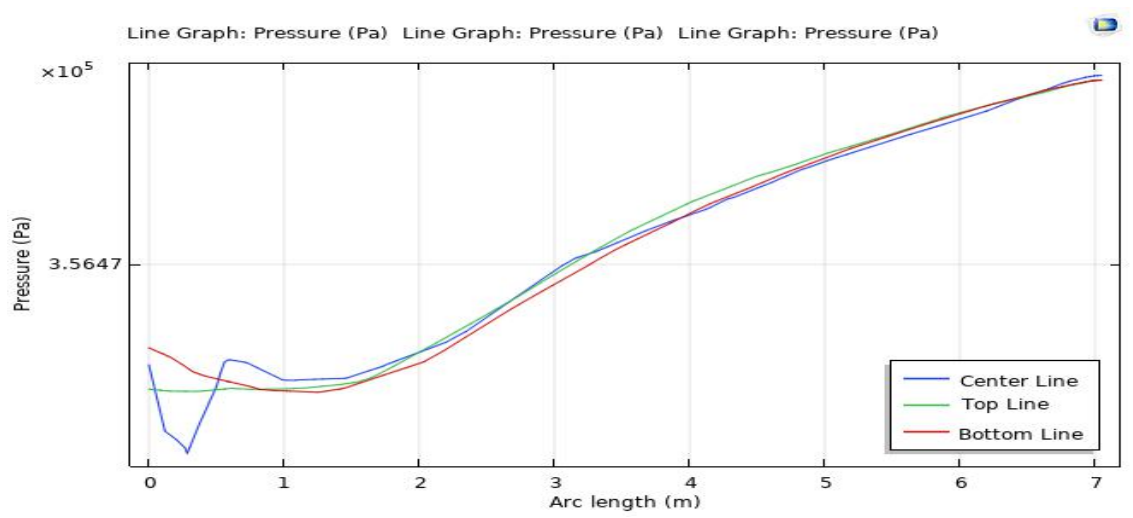

Figure 5. pressure at three cut-lines along the altitude of the cylinder

It is shown that the pressure near the end of the injection pipe is the smallest, and it grows as we move to the opposite end of the tank with very small variation.

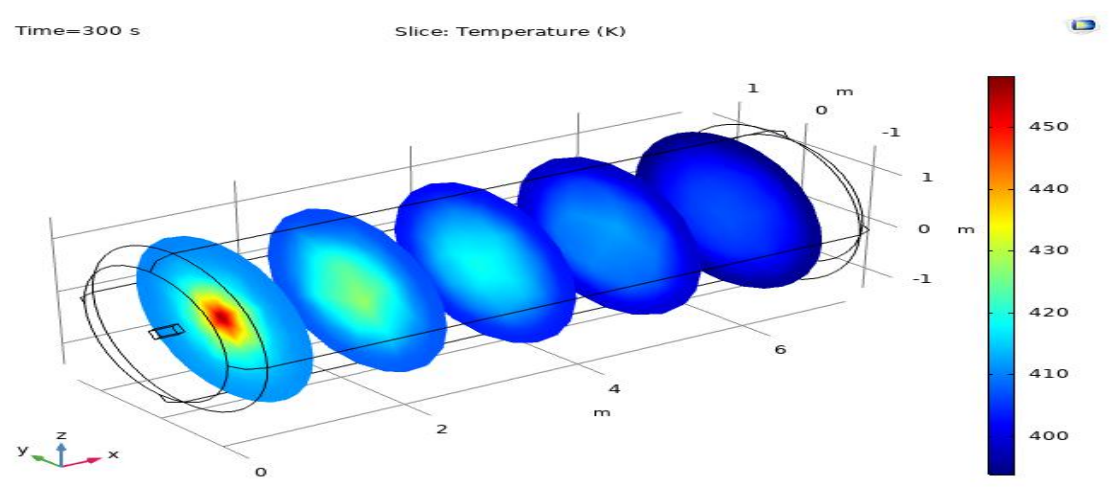

Figure 6. Temperature field in 5 planes in different positions

Figure 6 shows the temperature field in 5 planes in different positions along the tank, separated by the same distance. It can be seen that the temperature is uniformly distributed within the tank and near the injection pipe it has a higher temperature.

\section{CONCLUSION}

Results show various conditions of pressure fields along with temperature in different locations inside the storage tube produced through the inflow of hydrogen gas. Zero-dimensional modeling has also been carried out to compare with the results. This theoretical model shows qualitative agreement with the COMSOL results. It was assumed that mathematical model follows the ideal-gas law. By looking carefully at figure 5, although the mathematical and simulation model are passing different pathes, they cross each other eventually at the final pressure point. It is worth pointing out that the tank consisting of a $7.3 \mathrm{~m}$ long 
cylinder with a 50 bar capacity needs more time to be completely filled, hence, it would require a longer simulation time. Nevertheless, as long as mathematical and computational models support each other, we can use them as models, for example, to develop a set of controllers for the valve to adjust the pressure of the storage tank as desired.

\section{ACKNOWLEDGMENT}

The authors would like to thank the National Science Foundation (NSF) and specifically the Energy, Power, Control and Networks (EPCN) program for their valuable ongoing support in this research within the framework of grant ECCS-1809182 'Collaborative Research: Design and Control of Networked Offshore Hydrokinetic Power-Plants with Energy Storage’.

\section{NOMENCLATURE}

$\begin{array}{ll}\mathrm{m} & \mathrm{Kg} \\ \dot{m}_{i} & \mathrm{Kg} / \mathrm{s} \\ \dot{m}_{0} & \mathrm{Kg} / \mathrm{s} \\ \mathrm{t} & \mathrm{s} \\ \mathrm{U} & \mathrm{J} \\ \dot{Q} & \mathrm{~J} / \mathrm{s} \\ \dot{W} & \mathrm{~J} / \mathrm{s} \\ \mathrm{h} & \mathrm{J} / \mathrm{Kg} \\ c_{v} & \mathrm{~J} / \mathrm{Kg}-\mathrm{K} \\ \mathrm{T} & \mathrm{K} \\ \mathrm{P} & \mathrm{Pa} \\ \mathrm{v} & \mathrm{m} / \mathrm{s} \\ \mathrm{D} & \mathrm{m} \\ & \end{array}$

\section{REFERENCES}

[1] Amdreas Zuttel, Hydrogen Storage Method, In the science of nature. 91(4):157-72 - April 2004 TY - JOUR 
[2] U.S. Dept of Energy (http://chfcc.org/hydrogen-fuel-cells/about-hydrogen/hydrogen-properties/), Connecticut HydrogenFuel cell coalition(Source: U.S. Dept of Energy)

[3] https://www1.eere.energy.gov/hydrogenandfuelcells/tech validation/pdfs/fcm01r0.pdf, Collage of Desert, Deccember 2001

[4] Prof. S. Srinivasa Murthy, Chairman, Sub- Committee on Hydrogen Storage and Applications other than Transportation, REPORT ON HYDROGEN STORAGE AND APPLICATIONS OTHER THAN TRANSPORTATION, Sub-Committee on Hydrogen Storage and Applications Other than Transportation of the Steering Committee on Hydrogen Energy and Fuel Cells Ministry of New and Renewable Energy, Government of India, New Delhi ,February 2016.

[5] Etienne Rivard, Michel Trudeau and Karim Zaghib. Hydrogen Storage for Mobility: A Review, Published online 2019 Jun 19. doi: $\underline{10.3390 / \mathrm{ma12121973}}$

[6] Joakim Andersson, Stefan Gronkvist, Large-scale storage of hydrogen, International journal of hydrogen energy volume 44, issues 23, 3 May 2019, Pages 11901-11919

[7] Von Helmolt R, Eberle U, Fuel cell vehicles: status 2007. Journal of Power Sources 2007;165:833-843.

[8] Sofoklis S. Makridis, Hydrogen Storage and Compression, Chapter 1, CH001 18 June 2016; 11:30:20

[9] European Integrated Hydrogen Project EIHP ,www.eihp.org.

[10] E. TZIMAS, C. FILIOU, S.D. PETEVES and J.-B. VEYRET Petten, The Netherlands, Hydrogen Storage: State of the Art and Future Perspective., EUR 20995 EN, Luxembourg: Office for Official Publications of the European Communities, 2003, ISBN 92-894-6950-1, Printed in The Netherlands, Institute for Energy - JRC IE, PR\&Communication

[11] Bulk hydrogen storage, Presentation at the Strategic Directions for Hydrogen Delivery Workshop, Crystal City, USA, May 7-8, 2003.

[12] G. Thomas et. al., Hydrogen storage-Overview, ibid.

[13] R. Harris, Hydrogen storage: A critical technology for the future, presentation at the St. Andrews University, UK, July 3 , 2003.

[14] A. Zuttel, Materials for hydrogen storage, Materials Today, September 2003, pp. 24-33.

[15] COMSOL's CFD Module User's Guide (Version 5.4)

[16] https://www.comsol.eu/blogs/modeling-aeroacoustics-with-the-linearized-navier-stokes-equations/ Online source

[17] Yunus A. Cengel, John M. Cimbala, Fluid Mechanics Fundamentals and Applications, Second edition, Mc Graw Hill, Page 340. 